\title{
CONTINUOUS MAPS OF THE INTERVAL WHOSE PERIODIC POINTS FORM A CLOSED SET
}

\author{
ETHAN M. COVEN AND G. A. HEDLUND \\ Dedicated to the memory of Rufus Bowen
}

\begin{abstract}
We show that for a continuous map of a closed interval to itself, if the set of periodic points is closed, then every recurrent point is periodic. If, furthermore, the set of least periods of the periodic points is finite, then every nonwandering point is periodic. This answers a question of L. Block [Proc. Amer. Math. Soc. 67 (1977), 357-360].
\end{abstract}

Introduction. In [1] and [2], L. Block studied continuous maps of a closed interval to itself with finitely many periodic points. He showed [1, Theorem B] that if, in addition, every periodic point is fixed, then every nonwandering point is periodic, and [2, Theorem A] that the same conclusion holds if the set of nonwandering points is finite. In [1] he raised the question of whether this conclusion follows just from the assumption that the set of periodic points is finite.

The main result of this paper is the following, which answers Block's question in the affirmative.

THEOREM 4. For a continuous map of a closed interval to itself, if the set of least periods of the periodic points is finite, then every nonwandering point is periodic.

In the course of deriving this result, we show (Theorem 3) that Block's result of [1] is true without the assumption that there are only finitely many periodic points. We also show (Theorem 1) that if the set of periodic points is closed, then every recurrent point is periodic, and (Corollary to Theorem 2) that if the set of least periods of the periodic points is finite, then every orbit is asymptotic to a periodic orbit.

Notation and terminology. Throughout this paper, $f:[a, b] \rightarrow[a, b]$ will be continuous.

A point $x \in[a, b]$ is fixed provided that $f(x)=x ; x$ is periodic provided that there exists $n>1$ such that $f^{n}(x)=x$ (any such $n$ is called a period of $x$ ); $x$ is recurrent provided that for every neighborhood $U$ of $x$, there exists $n \geqslant 1$ such that $f^{n}(x) \in U ; x$ is nonwandering provided that for every neighborhood $U$ of $x$, there exists $n \geqslant 1$ such that $f^{n}(U) \cap U \neq \varnothing$. Let $F(f), P(f), R(f)$ and $N W(f)$ denote the sets of fixed, periodic, recurrent and nonwandering points, respectively. Each of

Received by the editors February 12, 1979 and, in revised form, May 26, 1979.

AMS (MOS) subject classifications (1970). Primary 54H20. 
these sets is invariant, i.e., $f$ maps each set to itself. Furthermore, $\varnothing \neq F(f) \subseteq P(f)$ $\subseteq R(f) \subseteq N W(f), F(f)$ and $N W(f)$ are closed, and for each $n \geqslant 1, P(f)=P\left(f^{n}\right)$ and [3] $R(f)=R\left(f^{n}\right)$.

Let $N P(f), N R(f)$ and $W(f)$ denote the sets of nonperiodic, nonrecurrent and wandering (i.e., not nonwandering) points, respectively.

Let $C \subseteq[a, b]$. We say that $f$ is completely positive on $C$ provided that $f^{n}(x)>x$ for all $x \in C$ and all $n \geqslant 1 ; f$ is completely negative on $C$ provided that $f^{n}(x)<x$ for all $x \in C$ and all $n \geqslant 1$.

Let $I, J \subseteq[a, b]$. We write $I<J$ provided that $x<y$ for all $x \in I$ and all $y \in J$.

By a basic neighborhood of $x \in(a, b)$ we mean an open interval $I$ such that $x \in I \subseteq(a, b)$. A basic neighborhood of $a$ is an interval of the form $[a, c)$ where $c<b$ and a basic neighborhood of $b$ is an interval of the form $(d, b]$ where $d>a$.

Results. If $P(f)$ is closed, then the components of $N P(f)$ are of the form $(p, q)$, $[a, q)$ or $(p, b]$ where $p, q \in P(f)$. Note that $P(f)$ is closed if $P(f)=F(f)$, or more generally, if the set of least periods of the periodic points is finite.

LEMMA 1. If $C$ is a component of $N P(f)$, then for each $n \geqslant 1$, either $f^{n}(x)>x$ for all $x \in C$ or $f^{n}(x)<x$ for all $x \in C$.

In particular, if $a \in C$, then $f$ is completely positive on $C$ and if $b \in C$, then $f$ is completely negative on $C$.

Proof. It suffices to consider the case where $C$ is an interval. Let $n \geqslant 1$ and suppose that there exist $x, y \in C$ such that $f^{n}(x)>x$ and $f^{n}(y)<y$. Then there exists $z \in C$ such that $f^{n}(z)=z$ and hence $P(f) \cap C \neq \varnothing$.

If $a \in C$, then $a \in N P(f)$ and $f^{n}(a)>a$ for all $n \geqslant 1$. Hence $f$ is completely positive on $C$. Similarly, if $b \in C$, then $f$ is completely negative on $C$.

LEMMA 2. If $C=(p, q)$ is a component of $N P(f)$ and $p$ and $q$ are fixed, then $f$ is either completely positive on $C$ or completely negative on $C$.

Proof. By Lemma 1, either $f(x)>x$ for all $x \in C$ or $f(x)<x$ for all $x \in C$. We assume the former.

Let $n \geqslant 1$. Since $C$ is a component of $N P(f)=N P\left(f^{n}\right)$, it follows from Lemma 1 that either $f^{n}(x)>x$ for all $x \in C$ or $f^{n}(x)<x$ for all $x \in C$. But $f^{i}(p)=p$ for all $i \geqslant 1$, so if $x \in C$ is sufficiently close to $p$, then $x<f(x)<\cdots<f^{n}(x)$. Therefore $f^{n}(x)>x$ for all $x \in C$. Since $n$ was arbitrary, $f$ is completely positive on C.

Similarly, if $f(x)<x$ for all $x \in C$, then $f$ is completely negative on $C$.

An immediate consequence of Lemmas 1 and 2 is the following.

LeMMA 3. If $P(f)=F(f)$ and $C$ is a component of $N P(f)$, then $f$ is either completely positive on $C$ or completely negative on $C$.

THEOREM 1. If $f:[a, b] \rightarrow[a, b]$ is continuous and $P(f)$ is closed, then $R(f)=$ $P(f)$. 
Proof. It suffices to show that $N P(f) \subseteq N R(f)$. Let $x \in N P(f)$ and let $C$ be the component of $N P(f)$ containing $x$. Since $P(f)$ is closed, $C$ is a neighborhood of $x$.

Suppose that $a \in C$. We may assume that $f^{K}(x) \in C$ for some $K>1$, for otherwise there is nothing to prove. By Lemma $1, f$ and hence $f^{K}$ is completely positive on $C$. Thus for every $n \geqslant 2, f^{n K}(x)=f^{(n-1) K}\left(f^{K}(x)\right)>f^{K}(x)$, so letting $I=\left[a, f^{K}(x)\right)$, we have $f^{n K}(x) \notin I$ for all $n \geqslant 1$. Thus $x \in N R\left(f^{K}\right)$. By [3], $N R\left(f^{K}\right)=N R(f)$ and so $x \in N R(f)$. Similarly, if $b \in C$, then $x \in N R(f)$.

If $C=(p, q)$ where $p, q \in C$, let $M$ be a common period of $p$ and $q$. Then $p, q \in F\left(f^{M}\right)$ and hence by Lemma $2, f^{M}$ is either completely positive on $C$ or completely negative on $C$. In either case an argument similar to the one in the preceding paragraph shows that $x \in N R(f)$.

THEOREM 2. If $f:[a, b] \rightarrow[a, b]$ is continuous and $P(f)=F(f)$, then for every $x \in[a, b]$, there exists $p \in F(f)$ such that $f^{n}(x) \rightarrow p$.

Proof. If $f^{n}(x) \rightarrow p$, then $p \in F(f)$, for $f^{n+1}(x) \rightarrow f(p)$ and $f^{n+1}(x) \rightarrow p$. Therefore it suffices to show that $\left\{f^{n}(x)\right\}$ converges for every $x \in[a, b]$.

If $f^{n}(x) \in P(f)$ for some $n \geqslant 0$, the result is trivial. Suppose that $f^{n}(x) \in N P(f)$ for all $n \geqslant 0$. Let $C_{n}$ be the component of $N P(f)$ containing $f^{n}(x)$. Let $\xi_{n}=+1$ if $f$ is completely positive on $C_{n}$ and let $\xi_{n}=-1$ if $f$ is completely negative on $C_{n}$.

If there exists $N \geqslant 0$ such that $\xi_{n}=+1$ for all $n \geqslant N$, then $f^{N}(x)<f^{N+1}(x)$ $<\ldots$ and so $\left\{f^{n}(x)\right\}$ converges. Similarly, if $\xi_{n}=-1$ for all $n \geqslant N$, then $\left\{f^{n}(x)\right\}$ converges.

Suppose that both +1 and -1 appear infinitely often in the sequence $\left\{\xi_{0}, \xi_{1}, \ldots\right\}$. Let $\mathscr{P}=\left\{n \geqslant 0 \mid \xi_{n}=+1\right\}=\left\{p_{1}<p_{2}<\ldots\right\}$ and $\mathscr{R}=\{n>0 \mid$ $\left.\xi_{n}=-1\right\}=\left\{m_{1}<m_{2}<\ldots\right\}$. Then $\left\{f^{p_{i}}(x)\right\}$ is increasing and $\left\{f^{m_{1}}(x)\right\}$ is decreasing and hence there exist $p, q \in[a, b]$ such that $f^{p_{i}}(x) \rightarrow p$ and $f^{m_{1}}(x) \rightarrow q$. There is a subsequence $\left\{k_{i}\right\}$ of the positive integers such that $k_{i} \in \mathscr{P}$ and $k_{i}+1 \in \mathfrak{R}$. Then $f^{k_{i}+1}(x) \rightarrow f(p)$ and $f^{k_{i}+1}(x) \rightarrow q$, and hence $f(p)=q$. Similarly, $f(q)=p$. Therefore $p \in P(f)=F(f)$ and hence $p=q$.

Corollary. If $f:[a, b] \rightarrow[a, b]$ is continuous and the set of least periods of the periodic points is finite, then for every $x \in[a, b]$, there exists $p \in P(f)$ such that $\left|f^{n}(x)-f^{n}(p)\right| \rightarrow 0$.

Proof. Apply Theorem 2 to $f^{N}$ where $N$ is a common period of the periodic points.

LEMMa 4. Let $P(f)$ be closed and let $C$ and $D$ be components of $N P(f)$ such that $f$ is completely positive on both $C$ and $D$. If for some $n \geqslant 1, x \in C$ and $f^{n}(x) \in D$, then $x \in W(f)$.

A similar statement holds if $f$ is completely negative on both $C$ and $D$.

Proof. Since $x \in N P(f), x \neq f^{n}(x)$. Since $C$ and $D$ are intervals, there are disjoint basic neighborhoods $I$ of $x$ and $J$ of $f^{n}(x)$ such that $I \subseteq C, J \subseteq D$ and $f^{n}(I) \subseteq J$. Since $f$ is completely positive on $C, I<J$. 
To show that $x \in W(f)$, it suffices to show that $f^{n+i}(I) \cap I=\varnothing$ for all $i>1$. Let $y \in I$ and $i \geqslant 1$. Then $f^{n}(y) \in J \subseteq D$. Since $f$ is completely positive on $D$, $f^{n+i}(y)>f^{n}(y)$ and since $I<J, f^{n+i}(y) \notin I$.

THEOREM 3. If $f:[a, b] \rightarrow[a, b]$ is continuous and $P(f)=F(f)$, then $N W(f)=$ $P(f)$.

Proof. It suffices to show that $N P(f) \subseteq W(f)$. Let $x \in N P(f)$.

Suppose that $f^{n}(x) \in N P(f)$ for all $n \geqslant 0$. Let $C_{n}$ be the component of $N P(f)$ containing $f^{n}(x)$. By Lemma 3 , there exist $i>j \geqslant 0$ such that $f$ is either completely positive on both $C_{i}$ and $C_{j}$ or completely negative on both $C_{i}$ and $C_{j}$. Since $f^{j}(x) \in C_{j}$ and $f^{i-j}\left(f^{j}(x)\right) \in C_{i}, f^{j}(x) \in W(f)$ by Lemma 4 and hence $x \in W(f)$.

Suppose that $f^{m}(x) \in P(f)$ for some $m \geqslant 1$ and that $x \in N W(f)$. We may assume that $y=f^{m-1}(x) \in N P(f)$. Then $y \in N W(f) \cap N P(f)$ and $f(y) \in P(f)$. Let $C$ be the component of $N P(f)$ containing $y$. By Lemma 3,f is either completely positive on $C$ or completely negative on $C$. We assume the former.

There exist basic neighborhoods $I$ of $y$ and $J$ and $J^{\prime}$ of $f(y)$ such that $I \subseteq C$, $I \cap J=\varnothing, f(I) \subseteq J^{\prime} \subseteq J$ and $f\left(J^{\prime}\right) \subseteq J$. Then $I<J$. Since $y \in N W(f)$, there exist $z \in I$ and $k \geqslant 3$ such that $f^{k}(z) \in I$. We may assume that $f^{k-1}(z) \notin I$. Since $z, f^{k}(z) \in I$, it follows that $f(z), f^{k+1}(z) \in J^{\prime}$.

We show that

$$
f^{k+1}(z)<f^{k-1}(z)<f(z) .
$$

This will prove the theorem, for then $f^{k-1}(z) \in J^{\prime}$ and hence $f^{k}(z)=f\left(f^{k-1}(z)\right) \in$ $J$, which is impossible since $f^{k}(z) \in I$ and $I \cap J=\varnothing$.

To prove (*), note that $f^{k-1}(z)>z \in I$ and since $f^{k-1}(z) \notin I, f^{k-1}(z)>f^{k}(z)$. Then $f^{k-1}(z) \in N P(f)$, for otherwise $f^{k-1}(z) \in F(f)$ and hence $f^{k-1}(z)=f^{k}(z)$. Let $D$ be the component of $N P(f)$ containing $f^{k-1}(z)$. Since $f\left(f^{k-1}(z)\right)=f^{k}(z)<$ $f^{k-1}(z)$, it follows from Lemma 3 that $f$ is completely negative on $D$ and in particular, $f^{k+1}(z)=f^{2}\left(f^{k-1}(z)\right)<f^{k-1}(z)$.

As in the preceding paragraph, $f(z) \in N P(f)$. Let $E$ be the component of $N P(f)$ containing $f(z)$. Since $f^{k}(z) \in I, f(z) \in J$ and $I<J$, it follows that $f^{k-1}(f(z))=$ $f^{k}(z)<f(z)$. Hence by Lemma $3, f$ is completely negative on $E$ and thus $f^{k-1}(z)=$ $f^{k-2}(f(z))<f(z)$. This proves $(*)$ and hence the theorem.

The proof when $f$ is completely negative on $C$ is similar.

It follows from a theorem of Sarkovskii [4] (for an account in English, see [5]) that if the set of least periods of the periodic points is finite, then each such least period is a power of 2. (For a short proof of this consequence of Šarkovskir’s Theorem, see [2, Theorem B].)

Lemma 5. Let $N$ be the maximal least period of the periodic points, let $0<i<$ $N-1$ and let $C$ be a component of $N P(f)$. Then one of the following statements is true.

(1) $f^{n+i}(x)>x$ for all $x \in C$ and all $n \geq 0(n \geq 1$ if $i=0)$.

(2) $f^{n N+i}(x)<x$ for all $x \in C$ and all $n \geq 0(n \geq 1$ if $i=0)$. 
Proof. If $a \in C$ or $b \in C$, then the lemma is true by Lemma 1 . Suppose that $C=(p, q)$ where $p, q \in P(f)$. By Šarkovskî̌s Theorem, $N=2^{\nu}$ for some $\nu>0$. We prove the lemma by induction on $\nu$. The lemma is true for $\nu=0$ by Lemma 2. Suppose it is true for $\nu$; we prove it for $\nu+1$.

Let $2^{\nu+1}=2 N$ be the maximal least period of the periodic points and let $g=f^{2}$. Then $P(g)=P(f)$ and the maximal least period (under $g$ ) of the periodic points is $N$. By the inductive hypothesis, if $0 \leqslant i \leqslant N-1$, then one of the following statements is true.

(1') $g^{n+i}(x)>x$ for all $x \in C$ and all $n \geqslant 0(n \geqslant 1$ if $i=0)$.

$\left(2^{\prime}\right) g^{n+i}(x)<x$ for all $x \in C$ and all $n \geqslant 0(n \geqslant 1$ if $i=0)$.

Hence if $i$ is even and $0 \leqslant i<2 N-1$, then one of the following statements is true.

$\left(1^{\prime \prime}\right) f^{2 n N+i}(x)>x$ for all $x \in C$ and all $n \geqslant 0(n \geqslant 1$ if $i=0)$.

$\left(2^{\prime \prime}\right) f^{2 n N+i}(x)<x$ for all $x \in C$ and all $n \geqslant 0(n \geqslant 1$ if $i=0)$.

To complete the proof, it suffices to show that if $i$ is odd and $0<i<2 N-1$, then either $\left(1^{\prime \prime}\right)$ or $\left(2^{\prime \prime}\right)$ is true.

Suppose that $f^{i}(p)=p$ and $f^{i}(q)=q$. Then $i$ is a multiple of the least period of $p$, which is a power of 2 ; hence $i=1$. Therefore $p$ and $q$ are fixed points and hence by Lemma $2, f$ is either completely positive on $C$ or completely negative on $C$. Thus either $\left(1^{\prime \prime}\right)$ or $\left(2^{\prime \prime}\right)$ is true.

Suppose that $f^{i}(p)>p$ and let $n \geqslant 0$. Since $f^{2 n N+i}(p)=f^{i}(p), f^{2 n N+i}(p)>p$. If $x \in C$ is sufficiently close to $p$, then $f^{2 n N+i}(x)>x$. Hence by Lemma $1,\left(1^{\prime \prime}\right)$ is true. Similarly, if $f^{i}(p)<p$, then $\left(2^{\prime \prime}\right)$ is true. The proofs when $f^{i}(q) \neq q$ are similar.

LeMma 6. Let $N$ be the maximal least period of the periodic points and let $C=(p, q)$ be a component of $N P(f)$. Let $i \geqslant 0$ and suppose that $f^{n+i}(x)>x$ for all $x \in C$ and all $n \geqslant 0$ ( $n \geqslant 1$ if $i=0)$. If there exist $y \in C, m>0$ and $j, k>1$ such that $f^{m N+j}(y) \in C$ and $k \equiv i-j(\bmod N)$, then $f^{n N+k}(x)>x$ for all $x \in C$ and all $n>0$.

A similar statement is true if $f^{n N+i}(x)<x$ for all $x \in C$ and all $n>0(n>1$ if $i=0)$.

Proof. If the conclusion is false, it follows from Lemma 5 that $f^{n N+k}(x)<x$ for all $x \in C$ and all $n \geqslant 0$. Let $z=f^{m N+j}(y)$. By Theorem 2 applied to $f^{N}, f^{n N}(z) \rightarrow r$ $\in P\left(f^{N}\right)=P(f)$. Hence $f^{n N+k}(z) \rightarrow f^{k}(r) \in P(f)$. Since $f^{n N+k}(z)<z$ for all $n>0$ and $p$ is the largest periodic point less than $z$, we have $f^{k}(r)<p$.

Since $k+j \equiv i(\bmod N), k+j=i+h N$ for some integer $h$. Then $f^{n N+k}(z)=$ $f^{n N+k}\left(f^{m N+j}(y)\right)=f^{(m+n+h) N+i}(y)>y$ if $m+n+h>1$. It follows that $f^{k}(r) \geqslant y$ $>p$. This contradiction proves the lemma.

LEMMA 7. Let the set of least periods of the periodic points be finite and let $C$ be a component of $N P(f)$. If $N W(f) \cap W\left(f^{2}\right) \cap C \neq \varnothing$, then $f$ is either completely positive on $C$ or completely negative on $C$. 
Proof. If $a \in C$ or $b \in C$, then the lemma is true by Lemma 1. Suppose $C=(p, q)$ where $p, q \in P(f)$. Let $N$ be the maximal least period of the periodic points. Then the lemma is true if $N=1$ by Lemma 2, so we assume that $N>2$.

Let $x \in N W(f) \cap W\left(f^{2}\right) \cap C$. Since $x \in W\left(f^{2}\right)$, there is a basic neighborhood $I$ of $x$ such that $I \subseteq C$ and $f^{2 j}(I) \cap I=\varnothing$ for all $j>1$. Since $x \in N W(f)$, there exist $y \in I$ and $k \geqslant 1$ such that $f^{k}(y) \in I$. Then $k$ must be odd and we write $k=m N+j$ where $m \geqslant 0$ and $j \geqslant 1$ is odd.

Since $F\left(f^{N}\right)=P\left(f^{N}\right)=P(f)$, it follows from Lemma 2 applied to $f^{N}$ that $f^{N}$ is either completely positive on $C$ or completely negative on $C$. We assume the former.

For $0 \leqslant i \leqslant N-1$, define $k_{i}$ by $k_{0}=0, k_{i+1} \equiv k_{i}-j(\bmod N)$ and $0<k_{i}<$ $N-1$. In particular, $k_{1} \equiv k_{0}-j(\bmod N)$ and since $f^{n N+k_{o}}(z)=f^{n N}(z)>z$ for all $z \in C$ and all $n \geqslant 1$, it follows from Lemma 6 that $f^{n N+k_{1}}(z)>z$ for all $z \in C$ and all $n \geqslant 0$. By repeated applications of this argument, $f^{n N+k_{i}}(z)>z$ for all $z \in C$, all $n>0\left(n \geqslant 1\right.$ if $\left.k_{i}=0\right)$ and all $i, 0 \leqslant i \leqslant N-1$. But since $N$ is a power of 2 and $j$ is odd, $\left\{k_{i} \mid 0 \leqslant i \leqslant N-1\right\}$ is a complete set of residues modulo $N$. Hence $f$ is completely positive on $C$.

Similarly, if $f^{N}$ is completely negative on $C$, then so is $f$.

LEMMA 8. Let the set of least periods of the periodic points be finite and let $C$ be a component of $N P(f)$ such that $f$ is either completely positive on $C$ or completely negative on $C$. If $x \in C$ and $f(x) \in P(f)$, then $x \in W(f)$.

Proof. Let $N$ be the maximal least period of the periodic points. As in the proof of Theorem 3, the lemma is true if $N=1$, so we assume that $N \geqslant 2$.

Suppose that $f$ is completely positive on $C, x \in C, f(x) \in P(f)$ and $x \in N W(f)$. Then $x<f^{N}(x) \in P(f)=P\left(f^{N}\right)$. There are basic neighborhoods $I$ of $x$ and $J$ and $J^{\prime}$ of $f^{N}(x)$ such that $I \subseteq C, I<J$ (and in particular, $\left.I \cap J=\varnothing\right), f^{N}(I) \subseteq J^{\prime} \subseteq J$ and $f^{N}\left(J^{\prime}\right) \subseteq J$. Furthermore, we may assume that

$$
\begin{gathered}
f^{i}(I) \cap I=\varnothing \text { for } 1 \leqslant i \leqslant 2 N, \\
f^{n N}(I) \cap I=\varnothing \text { for all } n \geqslant 1 .
\end{gathered}
$$

Condition (1) can be satisfied because $f^{i}(x) \neq x$ for all $i \geqslant 1$ and condition (2) can be satisfied because, by Theorem 3, $x \in W\left(f^{N}\right)$.

Since $x \in N W(f)$, there exist $y \in I$ and $k \geqslant 1$ such that $f^{k}(y) \in I$. We may assume that $k$ is the least such integer. It follows from (1) that $k>2 N$ and hence we can write $k=m N+j$ where $m \geqslant 2$ and by (2), $1<j<N-1$. Since $y, f^{m+j}(y) \in I$, it follows that $f^{N}(y), f^{(m+1) N+j}(y) \in J^{\prime}$.

We show that

$$
f^{(m+1) N+j}(y)<f^{(m-1) N+j}(y)<f^{N}(y) .
$$

This will prove the lemma, for then $f^{(m-1) N+j}(y) \in J^{\prime}$ and hence $f^{m N+j}(y)=$ $f^{N}\left(f^{(m-1) N+j}(y)\right) \in J$, which is impossible since $f^{m+j}(y) \in I$ and $I \cap J=\varnothing$.

To prove (*), note that $f^{N}(y) \in N P(f)$, for otherwise $f^{m N+j}(y) \in P(f)$ and hence $P(f) \cap C \neq \varnothing$. Let $D$ be the component of $N P(f)$ containing $f^{N}(y)$. Since $I<J, f^{(m-1) N+j}\left(f^{N}(y)\right)=f^{m N+j}(y)<f^{N}(y)$, and it follows from Lemma 5 applied 
to $D$ that

$$
f^{n+j}\left(f^{N}(y)\right)<f^{N}(y) \text { for all } n>0 .
$$

Since $f$ is completely positive on $C$ and $y \in C, f^{(m-1) N+j}(y)>y$. Since $1<$ $(m-1) N+j \leqslant k-1, f^{(m-1) N+j}(y) \notin I$ and hence, since $f^{m+j}(y) \in I$, $f^{(m-1) N+j}(y)>f^{m N+j}(y)$. Then $f^{N}\left(f^{(m-1) N+j}(y)\right)=f^{m N+j}(y)<f^{(m-1) N+j}(y)$, $f^{(m-1) N+j}(y) \notin F\left(f^{N}\right)$ and it follows from Lemma 3 applied to $f^{N}$ that

$$
f^{n N}\left(f^{(m-1) N+j}(y)\right)<f^{(m-1) N+j}(y) \text { for all } n>1 .
$$

Setting $n=2$ in (4) and $n=m-2$ in (3), we get (*) and hence the lemma.

The proof when $f$ is completely negative on $C$ is similar.

TheOREM 4. If $f:[a, b] \rightarrow[a, b]$ is continuous and the set of least periods of the periodic points is finite, then $N W(f)=P(f)$.

Proof. It suffices to show that $N P(f) \cap N W(f)=\varnothing$.

By Šarkovskil's Theorem, the maximal least period of the periodic points is $2^{\nu}$ for some $\nu>0$. We prove the theorem by induction on $\nu$. The theorem is true for $\nu=0$ by Theorem 3. Suppose it is true for $\nu$; we prove it for $\nu+1$.

Let $2^{\nu+1}$ be the maximal least period of the periodic points and let $g=f^{2}$. Then $P(g)=P(f)$ and the maximal least period (under $g$ ) of the periodic points is $2^{\nu}$. By the inductive hypothesis, $N W(g)=P(g)$ and hence $N P(f)=N P(g)=W(g)=$ $W\left(f^{2}\right)$. We show that the assumption $N P(f) \cap N W(f) \neq \varnothing$ leads to a contradiction. Suppose that $x \in N P(f) \cap N W(f)$. Then $f^{n}(x) \in N W(f)$ for all $n>0$.

Suppose that $f^{n}(x) \in N P(f)$ for all $n>0$. Let $C_{n}$ be the component of $N P(f)$ containing $f^{n}(x)$. Thus for all $n \geqslant 0, f^{n}(x) \in N W(f) \cap W\left(f^{2}\right) \cap C_{n}$. By Lemma 7, there exist $i>j \geqslant 0$ such that $f$ is either completely positive on both $C_{i}$ and $C_{j}$ or completely negative on both $C_{i}$ and $C_{j}$. Then by Lemma $4, f^{j}(x) \in W(f)$ and hence $x \in W(f)$.

Suppose that $f^{m}(x) \in P(f)$ for some $m>1$. We may assume that $f^{m-1}(x) \in$ $N P(f)$. Let $C$ be the component of $N P(f)$ containing $f^{m-1}(x)$. Since $f^{m-1}(x) \in$ $N W(f) \cap W\left(f^{2}\right) \cap C$ and $f\left(f^{m-1}(x)\right)=f^{m}(x) \in P(f)$, it follows from Lemmas 7 and 8 that $f^{m-1}(x) \in W(f)$.

In either case we have a contradiction and hence $N P(f) \cap N W(f)=\varnothing$.

\section{REFERENCES}

1. L. Block, Mappings of the interval with finitely many periodic points have zero entropy, Proc. Amer. Math. Soc. 67 (1977), 357-360. MR 57 \# 7692.

2. Continuous maps of the interval with finite nonwandering set, Trans. Amer. Math. Soc. 240 (1978), 221-230. MR 57 \# 13887.

3. P. Erdös and A. H. Stone, Some remarks on almost periodic transformations, Bull. Amer. Math. Soc. 51 (1945), 126-130. MR 6, 165.

4. A. N. Sarkovskii, Co-existence of cycles of a continuous mapping of the line into itself, Ukrain. Mat. Ż. 16 (1964), 61-71. (Russian, English summary) MR 28 \#121.

5. P. Stefan, A theorem of Sarkouskii on the existence of periodic orbits of continuous endomorphisms of the real line, Comm. Math. Phys. 54 (1977), 237-248. MR 56 \#3898.

Department of Mathematics, Wesleyan University, Middletown, Connecticut 06457 